\title{
Quantifying shape changes of silicone breast implants in a murine model using in vivo micro-CT
} \footnotetext{
Emily E Anderson ${ }^{1}$, Egon Perilli ${ }^{1}$, Colin J Carati ${ }^{2}$, Karen J Reynolds ${ }^{1}$

${ }^{1}$ The Medical Device Research Institute, School of Computer Science, Engineering and Mathematics, Flinders University, SA, Australia.

${ }^{2}$ Department of Anatomy and Histology, School of Medicine, Flinders University, SA, Australia.
}

Word count: 2389 words. Number of pages: 17 pages. Number of figures: 5 figures. Number of tables: 2 tables.

Author for correspondence:

Prof Karen Reynolds

The Medical Device Research Institute, School of Computer Science, Engineering and Mathematics,

Flinders University

1284 South Rd, Clovelly Park, South Australia, 5042, Australia

Telephone Number: +618 82015190

Email address: karen.reynolds@flinders.edu.au

This is the author manuscript accepted for publication and has undergone full peer review but has not been through the copyediting, typesetting, pagination and proofreading process, which may lead to differences between this version and the Version record. Please cite this article as doi:10.1002/ jbm.b.33682. 


\section{Abstract}

A major complication of silicone breast implants is the formation of a capsule around the implant known as capsular contracture which results in the distortion of the implant. Recently, a mouse model for studying capsular contracture was examined using micro-computed tomography (micro-CT), however, only qualitative changes were reported. The aim of this study was to develop a quantitative method for comparing the shape changes of silicone implants using in vivo micro-CT.

Mice were bilaterally implanted with silicone implants and underwent ionizing radiation to induce capsular contracture. On day 28 post-surgery mice were examined in vivo using micro-CT. The reconstructed cross-section images were visually inspected to identify distortion. Measurements were taken in 2D and $3 D$ to quantify the shape of the implants in the normal $(n=11)$ and distorted $(n=5)$ groups.

The degree of anisotropy was significantly higher in the distorted implants in the transaxial view $(0.99$ vs $1.19, p=0.002)$ and the $y$-axis lengths were significantly shorter in the sagittal $(9.27 \mathrm{~mm}$ vs $8.55 \mathrm{~mm}, p=0.015)$ and coronal $(9.24 \mathrm{~mm}$ vs $8.76 \mathrm{~mm}, \mathrm{p}=0.031$ ) views, indicating a deviation from the circular cross-section and shortening of the long axis. The 3D analysis revealed a significantly lower average thickness (sphere-fitting method) in distorted implants $(6.86 \mathrm{~mm}$ vs $5.49 \mathrm{~mm}$, $p=0.002$ ), whereas the volume and surface area did not show significant changes.

Statistically significant differences between normal and distorted implants were found in $2 \mathrm{D}$ and $3 \mathrm{D}$ using distance measurements performed via micro-CT. This objective analysis method can be useful for a range of studies involving deformable implants using in vivo micro-CT. 
Keywords: shape, silicone, breast implant, micro-CT, capsular contracture, quantitative

Running title: Quantifying the shape of silicone breast implants in vivo
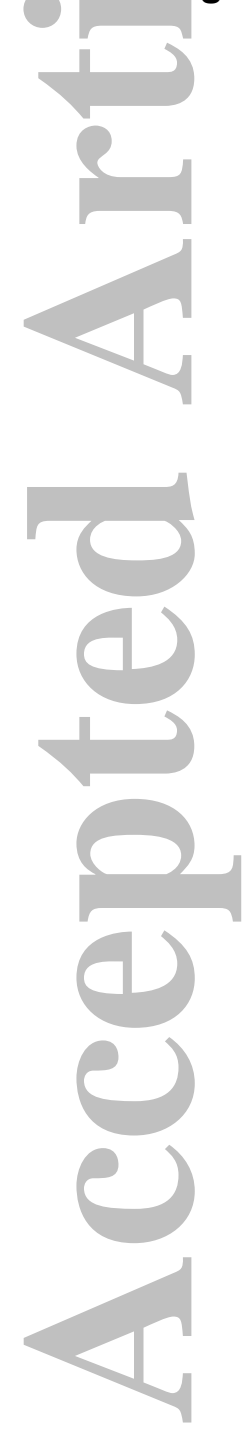

John Wiley \& Sons, Inc.

This article is protected by copyright. All rights reserved. 


\section{Introduction}

Silicone breast implants are commonly used for reconstructive surgeries or cosmetic purposes. Capsular contracture is a major complication resulting in connective tissue and associated cells forming a capsule around the implant ${ }^{1}$. This often leads to the distortion of the implant with associated hardening of the soft tissue area when manually assessed and is routinely treated by revision surgery ${ }^{2}$.

Animal models have been introduced to study capsular contracture ${ }^{3-6}$. Recently, capsular contracture studies have been performed in rat and mouse models, with the latter being monitored in live animals over time using in vivo micro-computed tomography (micro-CT) ${ }^{4}$, with a spatial resolution in the $10 \mu \mathrm{m}$ range. However, in these studies micro-CT was used only for qualitative observations, rather than quantitative measurements, to determine shape changes of the implants in vivo ${ }^{4}$. Hence, these studies were limited regarding the potential of micro-CT. Micro-CT enables quantitative measurements in $2 D$ and $3 D$ of the microarchitecture of anatomical parts of the animal, including bone and soft tissue ${ }^{7}$. A quantitative measurement method applied to micro-CT images in breast implant studies could enable the quantitative assessment of shape changes such as implant height or diameter, as well as numerical comparisons in these changes between treatments, quantifying their effect.

The aim of this study was to develop and demonstrate a straight forward quantitative analysis method applied to micro-CT images for assessing shape changes of silicone breast implants in vivo in mice. 


\section{Methods}

All experiments were undertaken in accordance with the Flinders University of South Australia Ethics Committee and the Southern Adelaide Local Health Network Animal Welfare Committee.

Surgery

The mice included here are part of a bigger ongoing study in our institute. Wild-type BalbC mice were maintained in the Flinders University School of Medicine Animal Facility and operated on at 6 to 8 weeks of age. On day 0 , animals were bilaterally implanted with two custom-made silicone gel implants (each 300mg, 10mm diameter, $9 \mathrm{~mm}$ height, nominal measurements provided by the manufacturer, Mentor Corporation, Santa Barbara, California) (Figure 1). The implants have a symmetrical geometry around their long axis (Figure 1 a) with a circular cross-section in the plane orthogonal to it (Figure $1 \mathrm{~b}$ ). For this study, 10 mice were used which resulted in 16 viable implants for analysis. Four implants were not available as one mouse received a single implant during surgery following complications with placement and three mice had one of their implants outside the scanning window during in vivo micro-CT scanning. Mice were anesthetised with $2 \%$ inhaled isoflurane and the surgical site was shaved and prepared with iodine solution. A $1 \mathrm{~cm}$ long transverse incision was made on the dorsal aspect of the mouse at the level of the sacral spine and two tunnels were dissected to the level of the rib cage ${ }^{4}$. The implant was placed over the ribs in a pocket under the skin and the panniculus carnosus muscle ${ }^{4}$. The incision was closed with tissue glue and surgical clips. On the same day, animals were allowed to recover and were transported to facilities for ionizing irradiation to induce 
capsular contracture (Department of Medical Physics, Royal Adelaide Hospital, Adelaide, SA, Australia).

\section{Irradiation with ionizing beam}

Mice were anesthetised with $2 \%$ inhaled isoflurane. A lead shield with the exposure area of the size of the implant was placed on the mouse, so that the adjacent tissue did not receive irradiation. Mice were positioned under the X-ray beam and irradiation was performed using a Gulmay D3150 superficial X-ray unit (Gulmay Limited, Surrey, United Kingdom). The settings were as follows: 10 Gy radiation dose, beam $50 \mathrm{kV}$, aluminium filter $1 \mathrm{~mm}$ in thickness, $3 \mathrm{~min} 31 \mathrm{sec}$ treatment time. This protocol was selected to induce capsular contracture around the implants while avoiding systemic effects of irradiation ${ }^{4}$. Animals were allowed to recover and were transported to their housing facilities.

In vivo micro-Computed Tomography (micro-CT) imaging

On day 28 post-surgery animals were transported to the facilities housing the microCT imaging equipment (Adelaide Microscopy, Adelaide, SA, Australia). Animals were anesthetised with $2 \%$ inhaled isoflurane and placed in the micro-CT scanner (Skyscan model 1076, Skyscan-Bruker, Kontich, Belgium). The micro-CT scanning window, $18 \mathrm{~mm}$ in length and $35 \mathrm{~mm}$ in width, was centred on the implants. The scanner settings were as follows: $8.65 \mu \mathrm{m}$ isotropic pixel size, source voltage $60 \mathrm{kV}$, current $100 \mu \mathrm{A}$, rotation step $0.8^{\circ}$, rotation over $180^{\circ}$, no frames averaging, aluminium filter $1 \mathrm{~mm}$-thick, total scanning time $20 \mathrm{~min}$ per mouse ${ }^{8}$. The cross-section images were reconstructed using a filtered back-projection algorithm (NRecon software, SkyScan-Bruker, Kontich, Belgium) and saved as 8-bit grey level images. 


\section{Quantitative Image Analysis}

For each implant, the micro-CT cross-section image dataset was realigned (software Dataviewer, Skyscan-Bruker, Kontich, Belgium), to have the long axis of the implant along the $y$-axis of the image in sagittal and in coronal views (Figure $2 \mathrm{a}, \mathrm{c}, \mathrm{d}, \mathrm{f}$ ), and the circular cross-section of the implant in the transaxial view (Figure $2 \mathrm{~b}, \mathrm{e}$ ). Implants were inspected visually through their micro-CT cross-section images (software Dataviewer, Skyscan-Bruker, Kontich, Belgium). Distortion was visually defined as those implants showing a distinct indentation on their surface. For the distorted implants, the transaxial images were realigned so that the elongated side of the (otherwise circular) implant was aligned along the $x$-axis in that view (horizontally), and the shorter along the y-axis (vertically), with the tip facing upwards (Figure 2 e).

Using the linear measurement tool (software Dataviewer, Skyscan-Bruker, Kontich, Belgium), for each view (sagittal, transaxial and coronal) two orthogonal distance measurements were taken (in $\mathrm{mm}$ ) over the cross-section images along the $\mathrm{x}$ and $\mathrm{y}$ axis respectively (Figure 2). For each view, the centre of the implant was defined by calculating the middle slice between the extreme boundaries of the implant (topbottom or left-right, respectively). The degree of anisotropy (DA) was calculated for each view, by dividing the longest axis measurement by the shortest axis measurement in that view ( $y / x$ for sagittal and coronal view, $x / y$ for transaxial view), which is an adaptation of the DA calculation proposed for trabecular bone ${ }^{9}$.

The stacks of images were then binarised (uniform threshold) to segment the implant as a solid (CT Analyser software, V1.14.4.1 Skyscan-Bruker, Kontich, Belgium). Over these images, the following 3D morphometric parameters were calculated: 
implant volume (measured in $\mathrm{mm}^{3}$ ) which corresponded to the sum of the voxels segmented as "implant", implant surface area (measured in $\mathrm{mm}^{2}$ ) which corresponded to the triangulated surface of the implant and calculated using the marching cube method ${ }^{10,11}$, and average model-independent thickness (measured in $\mathrm{mm}$ ) which was measured by the sphere-fitting method ${ }^{12,13}$ (CT Analyser software, V1.14.4.1 Skyscan-Bruker, Kontich, Belgium). The 3D models were visualised in Paraview V4.2.0 (Kitware, New York, USA).

Results are reported as median values and $1^{\text {st }}$ and $3^{\text {rd }}$ quartiles. A non-parametric Mann-Whitney U-test was undertaken to compare the measured parameters between the normal group and the distorted group (software IBM SPSS Statistics, New York, USA). Differences were deemed statistically significant for $p<0.05$.

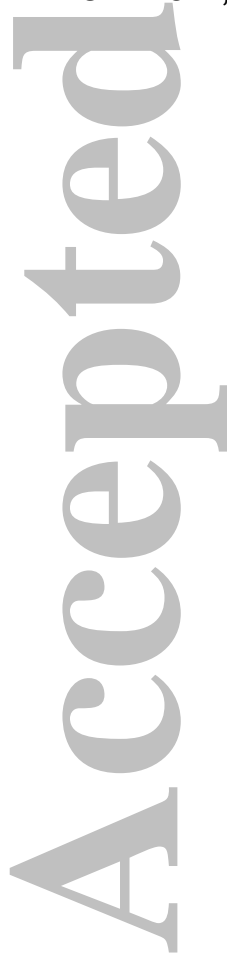

John Wiley \& Sons, Inc.

This article is protected by copyright. All rights reserved. 


\section{Results}

Implant distortion

Following micro-CT imaging on day 28 post-surgery, 5 implants showed distortion and 11 implants were identified as normal. Figure 3 shows examples of 3D models created from normal and distorted implants.

\section{$2 D$ distance analysis using micro-CT images}

2D measurements were taken in the sagittal, transaxial and coronal views (Figures $2,4)$ and results are shown in Table 1 . In the sagittal view, the distorted implants showed significantly shorter lengths along the $x(8.13[7.98,8.32] \mathrm{mm}$ vs $6.54[5.88$, $6.71] \mathrm{mm}, \mathrm{p}=0.009)$ and y $(9.27[8.96,9.55] \mathrm{mm}$ vs $8.55[8.31,8.82] \mathrm{mm}, \mathrm{p}=0.015)$ directions, with a trend to increase in the corresponding DA $(1.12[1.09,1.15]$ vs 1.35 $[1.24,1.49], p=0.062)$ compared to normal. In the transaxial view, the measured lengths were significantly lower for the distorted implants compared with normal implants in the y direction $(8.03[7.92,8.32] \mathrm{mm}$ vs $6.71[6.26,6.96] \mathrm{mm}, \mathrm{p}=0.002)$ but not in the $x$ direction $(p=0.610)$. The DA was significantly larger in the distorted implants $(0.99[0.98,1.00]$ vs $1.19[1.14,1.33], p=0.002)$. In the coronal view, the distorted implants compared to normal showed statistically significant lower lengths in the $y$ direction $(9.24[8.91,9.52] \mathrm{mm}$ vs $8.76[8.31,8.82] \mathrm{mm}, \mathrm{p}=0.031)$, however no differences in the $x$ direction $(p=0.069)$ and DA $(p=0.610)$.

\section{D analysis using micro-CT images}

The average model-independent thickness (Figure 5) was significantly smaller in distorted implants $(6.86[6.72,7.06] \mathrm{mm}$ vs. $5.49[5.49,5.80] \mathrm{mm}, \mathrm{p}=0.002)$ compared to normal implants. The measured implant volume, surface area and 
surface area to volume ratio did not show significant differences between normal and distorted implant groups (Table 2).
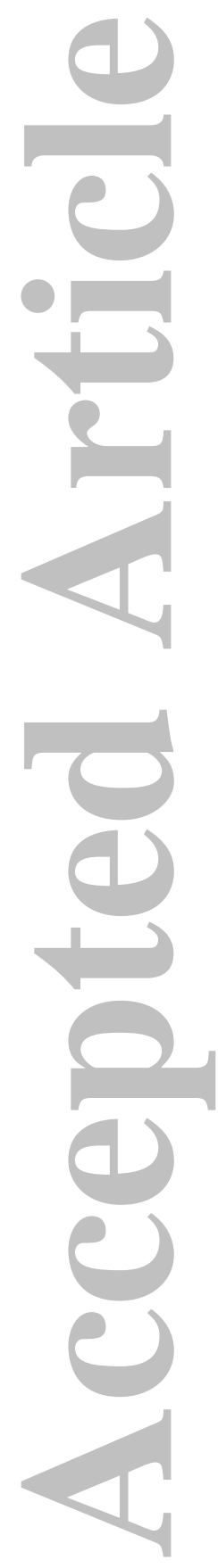

John Wiley \& Sons, Inc.

This article is protected by copyright. All rights reserved. 


\section{Discussion}

We have described a micro-CT method for analysis in both $2 \mathrm{D}$ and $3 \mathrm{D}$ of silicone implants in a murine model of capsular contracture, to provide a quantitative description of shape changes occurring in these implants. Statistically significant changes in implant height, diameter and degree of anisotropy, measured by microCT, were found.

Any change in the implant geometry results in a change in diameter and/or the length of the implant, which can be quantified. The distorted implants showed significantly shorter heights (Figure $4 \mathrm{~b}$ ), as well as smaller 3D thicknesses (Figure 5).

In addition to the linear measurements of the implant dimensions along the $\mathrm{x}$ and $\mathrm{y}$ axes (Figure 2), we have presented measurements of the degree of anisotropy (DA) (Figure 4). This has been adapted to silicone implants from previous work in other applications using micro-CT, such as bone studies, to identify the major axes of the investigated structure ${ }^{9}$. In the present case, any significant difference in DA values from those obtained in the normal implants would indicate implant distortion. Theoretically, there could be a change in the value of DA in any of the presented viewing planes (sagittal, transaxial, coronal, Figure 2) or only in some of them, depending on the view and how the distortion occurred. For example, for a thin circular object (a disc), as could be the case of a transaxial cross-section of an undistorted implant, the expected DA value is equivalent to 1 because there is no major axis in the disc plane and all lengths through the disc centre are equal to each other as well as equivalent to the disc diameter. In fact, in the transaxial view of this study, the normal implants showed a DA value of $0.99[0.98,1.00]$ which is a value very close to 1 , indicating a circular object in that plane (Figure $2 \mathrm{~B}$ ). However, the 
statistically significant higher value for the distorted implants group, DA = $1.19[1.14$, 1.33], indicates a change from the control group that is a statistically significant loss in circularity of the implant cross-section, resulting in visible distortion (Figures 2, 3 \& 4).

We also introduced the calculation of the 3D thickness of the silicone implant. It is a volume-based calculation ${ }^{12,13}$ and as such it is independent of the viewing plane (whether sagittal, transaxial, or coronal). It is based on a method that fits hypothetical maximal spheres inside the volume bound by the implant, with the average (volumeweighted) diameter of these spheres representing the average thickness of the examined object in 3D. The median thickness value $(6.86 \mathrm{~mm}$, Figure 5$)$ for the normal implants found in the present study corresponds to the median of the average diameters of the largest spheres (volume-weighted) that were confined within the exterior boundary of the implants. The statistically significant reduction in this value for the distorted implants $(5.49 \mathrm{~mm}, \mathrm{p}=0.002$, Figure 5$)$ indicates corresponding shape changes (Figure 3). This is in line with the significant change in DA in the transaxial plane found in this study (Figure 2, 4).

The values of implant surface area and volume did not show significant changes in the distorted implants compared to controls, suggesting that the amount of implant material was preserved within the implant and that no leaks or erosions occurred.

The presented method allows for an objective measure to quantify shape changes of the implant and can potentially be used to quantitatively compare the effects of pathologies and possible treatments. In a previous study by Katzel et al (2010), capsular contracture was studied in a mouse model using micro-CT. In that study, mice were implanted with silicone implants, treated with ionizing radiation to induce 
capsular contracture and then micro-CT scanned at days $0,21,28,35$ and 42 postsurgery. The analysis of their micro-CT images of the silicone implants was qualitative, concluding that animals showed capsular contracture as evidenced by a visual deformation in the implant. However, the shape changes were not quantified, which prevents any statistical comparison of quantitative effects of different treatments using micro-CT.

The quantitative methods that we have used are well-established in other fields of research using micro-CT such as in musculoskeletal research for diseases involving osteoporosis ${ }^{14}$, osteoarthritis ${ }^{15}$ and bone cancer ${ }^{16}$. These studies use the parameters of bone volume, bone surface area, trabecular thickness and degree of anisotropy, to assess features of interest such as the deterioration of trabecular and cortical bone in animal models and in excised bones from patients or cadavers ${ }^{17}$. Here we have shown that parameters used commonly in bone studies can be successfully adapted to study silicone breast implants in vivo in a mouse model. Moreover, this method could potentially be extended to other research models on deformable implants.

Some limitations of the quantitative method described here exist. First, we examined live animal micro-CT images at a single time-point on day 28 and we did not have access to images taken previously, as this study was part of a bigger ongoing study. Nonetheless, the study showed effective use of these micro-CT scans at the examined time-point. Second, in this study we evaluated samples of a single implant type. However, theoretically this method could be applied to other types of breast implants such as those which are textured ${ }^{5,18}$. Another limitation is the small sample size, in particular for the distorted group $(n=5)$ compared to the normal group $(n=11)$. 
To account for this, we used non-parametric statistics (Mann-Whitney U-test) instead of parametric statistics, for comparison between groups.

In conclusion, we have presented a method to quantitatively determine shape changes of silicone breast implants using in vivo micro-CT on mice. The micro-CT measurements in both $2 \mathrm{D}$ and $3 \mathrm{D}$ showed statistically significant differences between normal and distorted implants. This straight forward analysis method could be included in future studies of deformable implants using in vivo or ex vivo microCT.

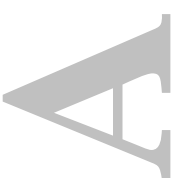

\section{Acknowledgements}

-The implants for this study were donated by the Mentor Corporation. The authors would like to thank Associate Professor William Scarlett, Buck's County Aesthetic Centre, PA, USA for organising the implants, Associate Professor Eva Bezak from the Department of Medical Physics, Royal Adelaide Hospital, Australia for administering the irradiation to the animals and Adelaide Microscopy for providing access to the micro-CT scanner. This work was supported by the South Australian Government's Department of Manufacturing, Innovation, Trade, Resources and Energy through the Medical Device Commercialisation Program.

\section{References}

1. Berry MG, Cucchiara V, Davies DM. Breast augmentation: Part II - adverse capsular contracture. Journal of Plastic Reconstructive and Aesthetic Surgery 2010;63(12):2098-2107. 
2. Araco A, Caruso R, Araco F, Overton J, Gravante G. Capsular Contractures: A Systematic Review. Plastic and Reconstructive Surgery 2009;124(6):18081819.

3. Brohim RM, Foresman PA, Grant GM, Merickel MB, Rodeheaver GT. Quantitative monitoring of capsular contraction around smooth and textured implants. Annals of Plastic Surgery 1993;30(5):424-434.

4. Katzel EB, Koltz PF, Tierney R, Williams JP, Awad HA, O'Keefe RJ, Langstein HN. A Novel Animal Model for Studying Silicone Gel-Related Capsular Contracture. Plastic and Reconstructive Surgery 2010;126(5):1482-1490.

5. Clugston PA, Perry LC, Hammond DC, Maxwell GP. A rat model for capsular contracture - the effects of surface texturing. Annals of Plastic Surgery $1994 ; 33(6): 595-599$.

6. Moreira M, Fagundes DJ, Simoes MD, Taha MO, Perez LMN, Bazotte RB. The effect of liposome-delivered prednisolone on collagen density, myofibroblasts, and fibrous capsule thickness around silicone breast implants in rats. Wound Repair and Regeneration 2010;18(4):417-425.

7. Perilli E, Cantley M, Marino V, Crotti TN, Smith MD, Haynes DR, Dharmapatni AASSK. Quantifying Not Only Bone Loss, but Also Soft Tissue Swelling, in a Murine Inflammatory Arthritis Model Using Micro-Computed Tomography. Scandinavian Journal of Immunology 2015;81(2):142-150.

8. Zawawi MSF, Perilli E, Stansborough RL, Marino V, Cantley MD, Xu J, Dharmapatni AASSK, Haynes DR, Gibson RJ, Crotti TN. Caffeic Acid Phenethyl Ester Abrogates Bone Resorption in a Murine Calvarial Model of Polyethylene Particle-Induced Osteolysis. Calcified Tissue International 2015:1-10. 
9. Goulet RW, Goldstein SA, Ciarelli MJ, Kuhn JL, Brown MB, Feldkamp LA. The relationship between the structural and orthogonal compressive properties of trabecular bone. Journal of Biomechanics 1994;27(4):375-389.

10. Ruegsegger P, Koller B, Muller R. A microtomographic system for the nondestructive evaluation of bone architecture. Calcified Tissue International $1996 ; 58(1): 24-29$.

11. Lorensen WE, Cline HE. Marching cubes: A high resolution 3D surface construction algorithm. SIGGRAPH Comput. Graph. 1987;21(4):163-169.

12. Hildebrand T, Ruegsegger P. A new method for the model-independent assessment of thickness in three-dimensional images. Journal of MicroscopyOxford 1997;185:67-75.

13. Perilli E, Baruffaldi F, Bisi MC, Cristofolini L, Cappello A. A physical phantom for the calibration of three-dimensional X-ray microtomography examination. Journal of Microscopy-Oxford 2006;222:124-134.

14. Perilli E, Le V, Ma B, Salmon P, Reynolds K, Fazzalari NL. Detecting early bone changes using in vivo micro-CT in ovariectomized, zoledronic acidtreated, and sham-operated rats. Osteoporosis International 2010;21(8):13711382.

15. Mohan G, Perilli E, Kuliwaba J, Parkinson I, Humphries J, Fazzalari N. Characterisation of temporal subchondral bone changes in a rat model of lowdose monosodium iodoacetate induced osteoarthritis: An in vivo micro-CT study. Osteoarthritis and Cartilage 2011;19:S61-S62.

16. Labrinidis A, Hay S, Liapis V, Findlay DM, Evdokiou A. Zoledronic acid protects against osteosarcoma-induced bone destruction but lacks efficacy 
against pulmonary metastases in a syngeneic rat model. International Journal of Cancer 2010;127(2):345-354.

17. Tassani S, Particelli F, Perilli E, Traina F, Baruffaldi F, Viceconti M. Dependence of trabecular structure on bone quantity: A comparison between osteoarthritic and non-pathological bone. Clinical Biomechanics $2011 ; 26(6): 632-639$.

18. Abramo AC, De Oliveira VR, Ledo-Silva MC, De Oliveira EL. How TextureInducing Contraction Vectors Affect the Fibrous Capsule Shrinkage Around Breasts Implants? Aesthetic Plastic Surgery 2010;34(5):555-560.

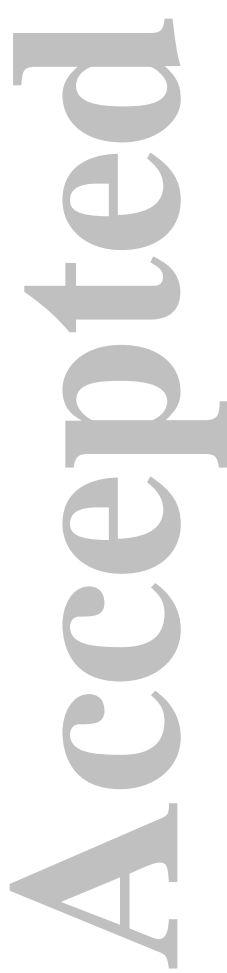

John Wiley \& Sons, Inc.

This article is protected by copyright. All rights reserved. 


\section{Figure Legends}

Figure 1: Images of the silicone implants ex vivo. (a) Side profile of a normal implant depicting the tip and base positions. The implant is symmetrical around the midline. (b) Top-down view of a normal implant showing the circular profile.

Figure 2: Reconstructed micro-CT cross-section images of a normal (a-c) and distorted (d-f) implant as shown in the sagittal $(a, d)$ transaxial $(b, e)$ and coronal $(c, f)$ planes. For each view, dashed arrows indicate the distance measurements taken in the $x$ direction, solid arrows in the $y$ direction. The deformation is evident in the sagittal and transaxial views of the distorted implant (shown by a *). Scale bar $=$ $1 \mathrm{~mm}$.

Figure 3: 3D micro-CT models of the implants obtained from in vivo scans in five mice $(8.65 \mu \mathrm{m}$ pixel size). Top row: five normal implants, bottom row: five distorted implants. Arrows point to the distortion (indent).

Figure 4: Box plot, reporting median values and first and third quartiles of the measurements made using micro-CT. Whiskers represent the maximum and minimum values. (a) Distance measurements taken along the x-axis (See figure 2) in the sagittal, transaxial and coronal views, for normal and distorted implants. (b) Distance measurements taken along the $y$ axis in the three views. There was a significant decrease in all views. (c) The degree of anisotropy calculated for the 
sagittal $(D A=y / x)$, transaxial $(D A=x / y)$ and coronal $(D A=y / x)$ views. ${ }^{*} p<0.05$, ${ }^{* *} p<0.01$, Mann-Whitney U-test.

Figure 5: Box plot, reporting median values and first and third quartiles of 3Dthickness measurements made using micro-CT. Whiskers represent the maximum and minimum values. There was a significant decrease in the 3D-thickness of distorted implants compared with normal implants. ${ }^{* *} p<0.01$, Mann-Whitney U-test.
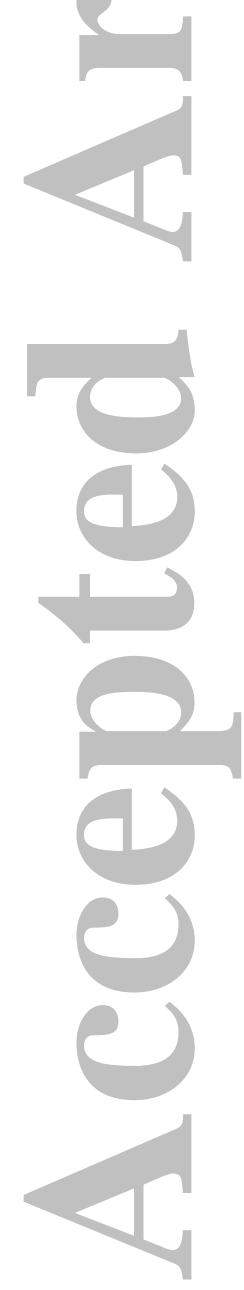

John Wiley \& Sons, Inc.

This article is protected by copyright. All rights reserved. 


\section{Tables}

Table 1: 2D distance measurements and degree of anisotropy (DA) for normal and distorted implants.

\begin{tabular}{cccc} 
Parameter & $\begin{array}{c}\text { Normal } \\
\text { Median [Q1, Q3] }\end{array}$ & $\begin{array}{c}\text { Distorted } \\
\text { Median [Q1, Q3] }\end{array}$ & P Value \\
\hline Sagittal & & & \\
$\mathrm{x}(\mathrm{mm})$ & $8.13[7.98,8.32]$ & $6.54[5.88,6.71]$ & 0.009 \\
$\mathrm{y}(\mathrm{mm})$ & $9.27[8.96,9.55]$ & $8.55[8.31,8.82]$ & 0.015 \\
$\mathrm{DA}=\mathrm{y} / \mathrm{x}$ & $1.12[1.09,1.15]$ & $1.35[1.24,1.49]$ & 0.062 \\
Transaxial & & & \\
\hline $\mathrm{x}(\mathrm{mm})$ & $7.96[7.87,8.18]$ & $8.13[8.10,8.27]$ & 0.610 \\
$\mathrm{y}(\mathrm{mm})$ & $8.03[7.92,8.32]$ & $6.71[6.26,6.96]$ & 0.002 \\
$\mathrm{DA}=\mathrm{x} / \mathrm{y}$ & $0.99[0.98,1.00]$ & $1.19[1.14,1.33]$ & 0.002 \\
Coronal & & & \\
$\mathrm{x}(\mathrm{mm})$ & $7.96[7.86,8.10]$ & $7.72[6.47,7.89]$ & 0.069 \\
$\mathrm{y}(\mathrm{mm})$ & $9.24[8.91,9.52]$ & $8.76[8.31,8.82]$ & 0.031 \\
$\mathrm{DA}=\mathrm{y} / \mathrm{x}$ & $1.15[1.12,1.18]$ & $1.16[1.12,1.28]$ & 0.610 \\
\hline
\end{tabular}

Q1: 1st Quartile; Q3: 3rd Quartile 
Table 2: 3D parameters for normal and distorted implants.

\begin{tabular}{lccc} 
Parameter & $\begin{array}{c}\text { Normal } \\
\text { Median [Q1, Q3] }\end{array}$ & $\begin{array}{c}\text { Distorted } \\
\text { Median [Q1, Q3] }\end{array}$ & P Value \\
\hline Thickness $(\mathrm{mm})$ & $6.86[6.72,7.06]$ & $5.49[5.49,5.80]$ & 0.002 \\
Surface $\left(\mathrm{mm}^{2}\right)$ & $393[380,407]$ & $382[369,383]$ & 0.096 \\
Volume $\left(\mathrm{mm}^{3}\right)$ & $361[340,366]$ & $333[328,342]$ & 0.072 \\
Surface/Volume & $1.12[1.10,1.14]$ & $1.12[1.10,1.15]$ & 0.650 \\
Ratio (1/mm) & & & \\
& & & \\
& & & \\
\hline & & & \\
& & \\
\end{tabular}

John Wiley \& Sons, Inc.

This article is protected by copyright. All rights reserved. 

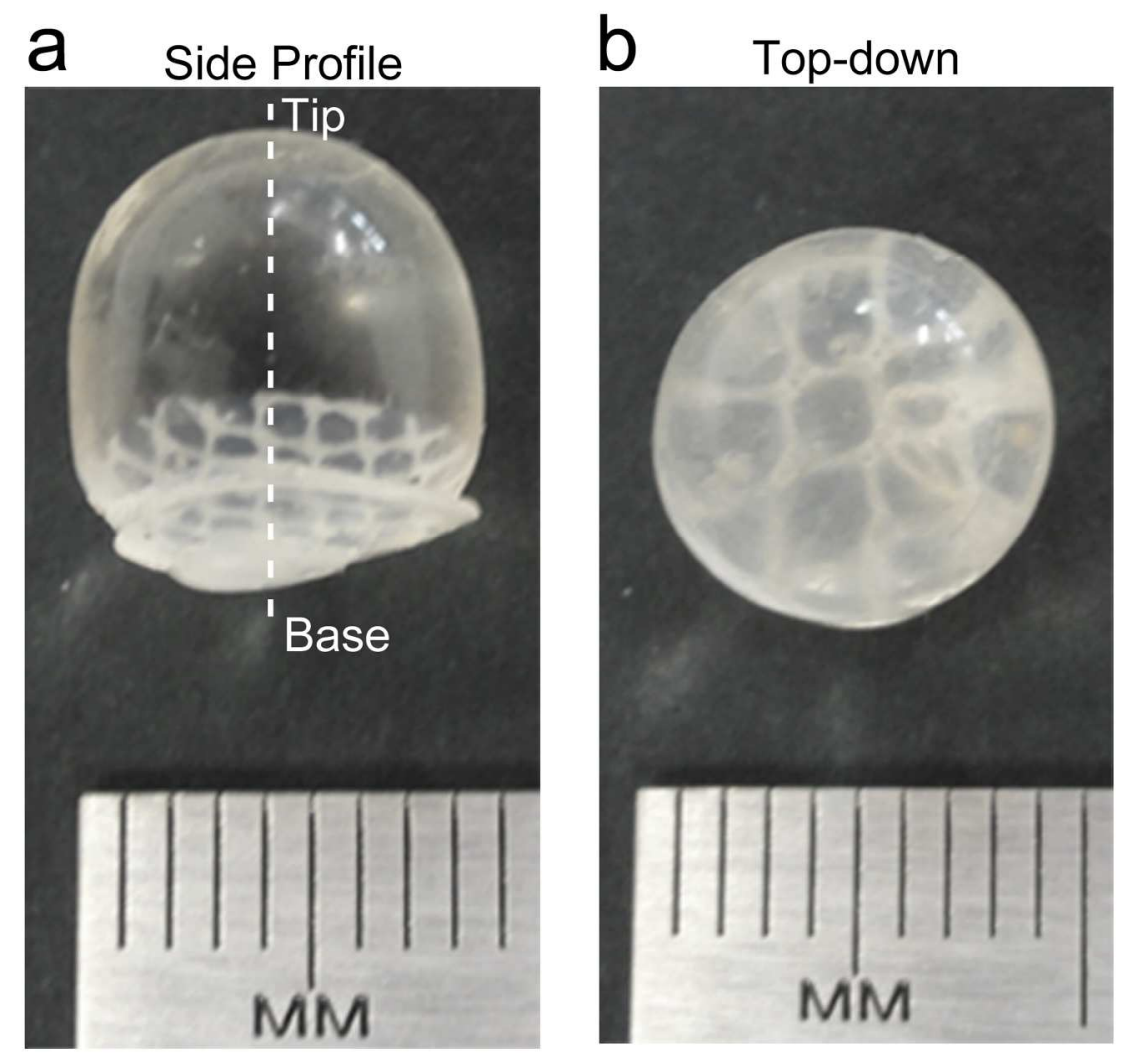

Figure 1: Images of the silicone implants ex vivo. (a) Side profile of a normal implant depicting the tip and base positions. The implant is symmetrical around the midline. (b) Top-down view of a normal implant showing the circular profile.

$168 \times 144 \mathrm{~mm}(300 \times 300 \mathrm{DPI})$

John Wiley \& Sons, Inc.

This article is protected by copyright. All rights reserved. 

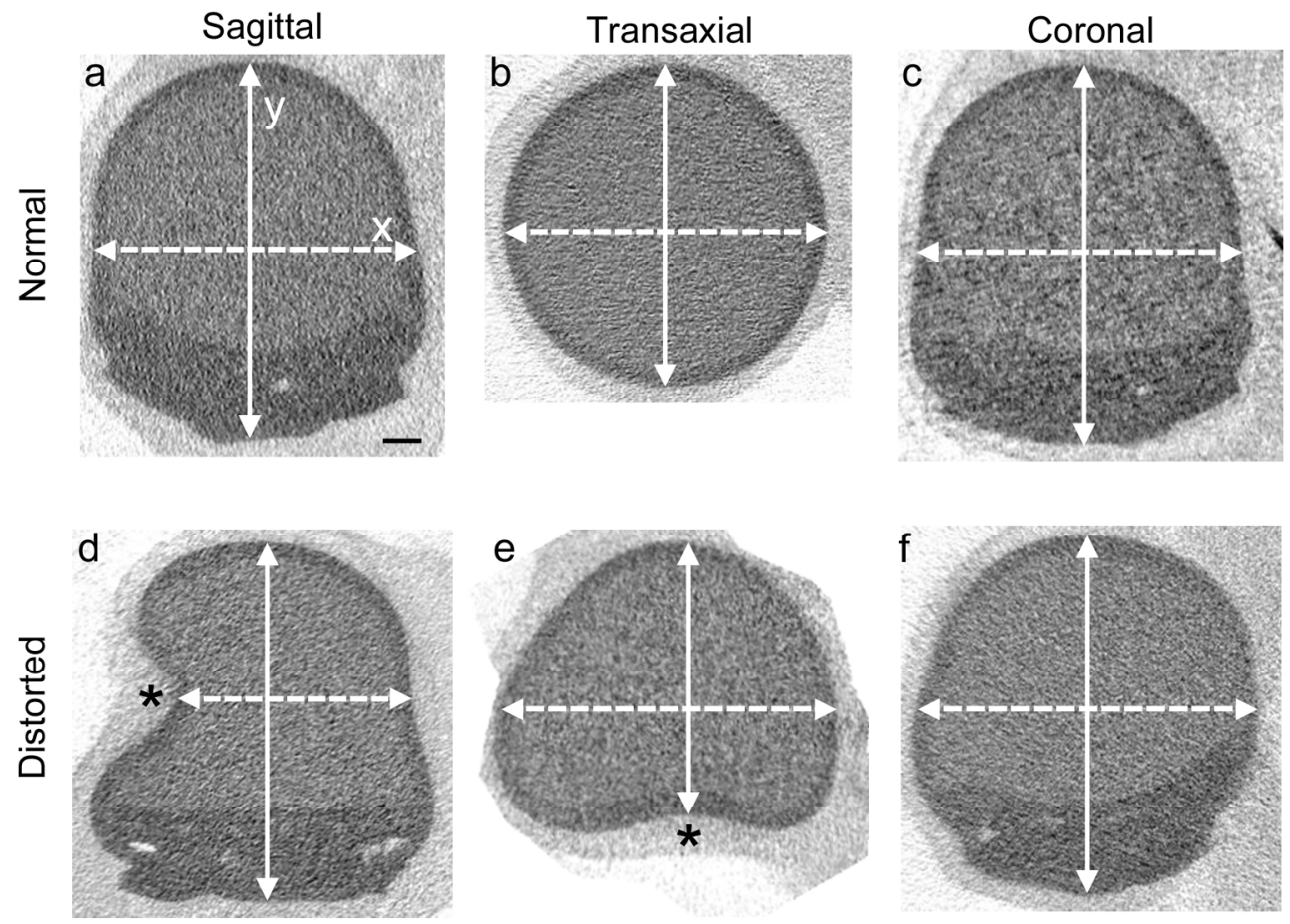

Figure 2: Reconstructed micro-CT cross-section images of a normal (a-c) and distorted (d-f) implant as shown in the sagittal $(a, d)$ transaxial $(b, e)$ and coronal $(c, f)$ planes. For each view, dashed arrows indicate the distance measurements taken in the $x$ direction, solid arrows in the $y$ direction. The deformation is evident in the sagittal and transaxial views of the distorted implant (shown by $a^{*}$ ). Scale bar $=1 \mathrm{~mm}$.

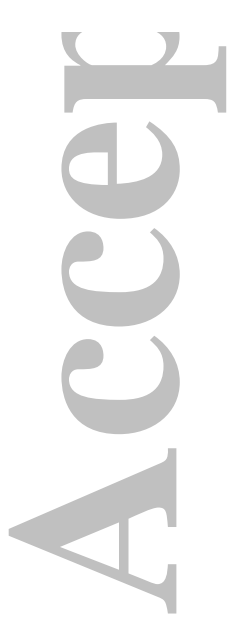
$168 \times 123 \mathrm{~mm}(300 \times 300 \mathrm{DPI})$ 


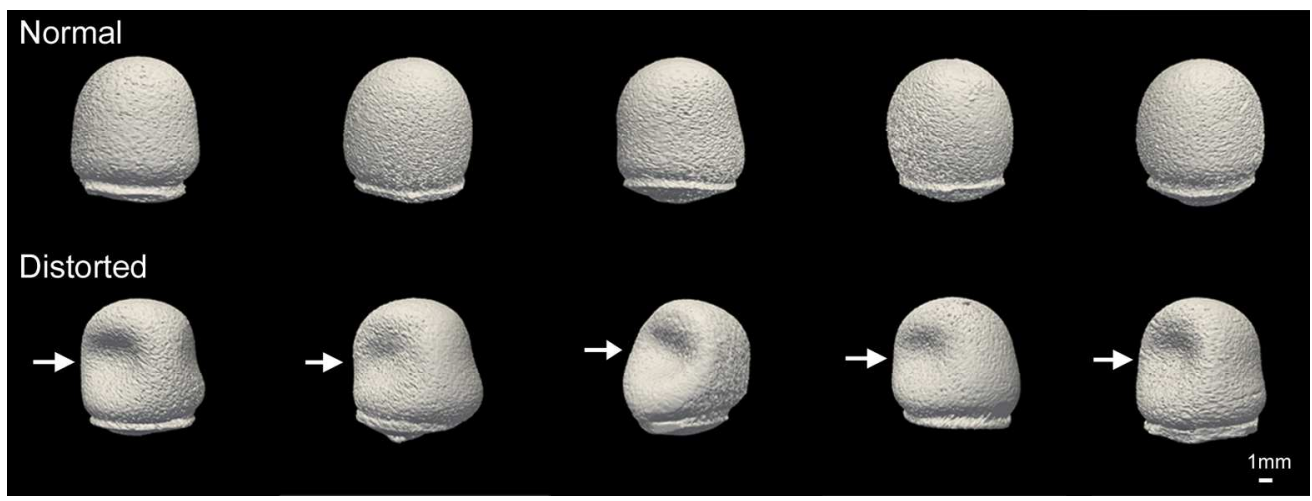

Figure 3: 3D micro-CT models of the implants obtained from in vivo scans in five mice $(8.65 \mu \mathrm{m}$ pixel size). Top row: five normal implants, bottom row: five distorted implants. Arrows point to the distortion (indent).

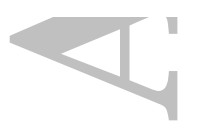
$168 \times 62 \mathrm{~mm}(300 \times 300 \mathrm{DPI})$

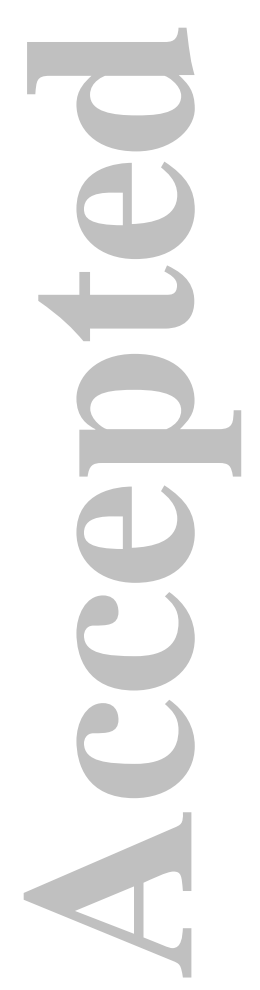

John Wiley \& Sons, Inc.

This article is protected by copyright. All rights reserved. 
A

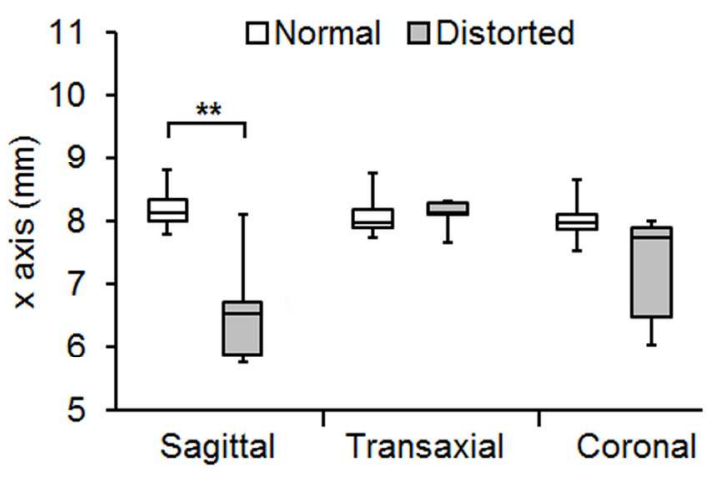

B

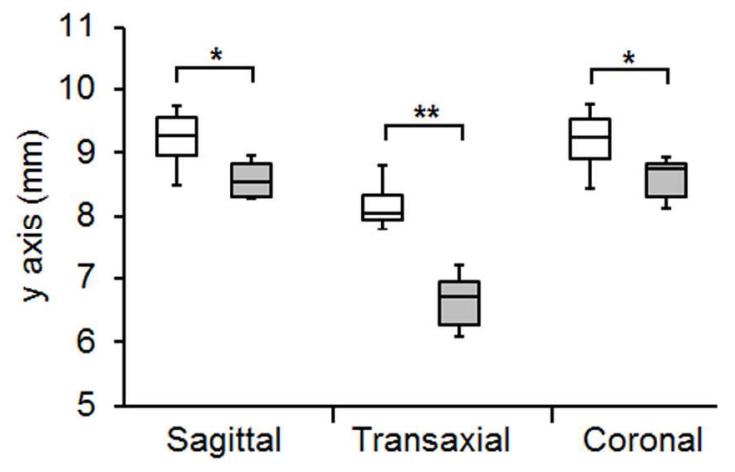

C

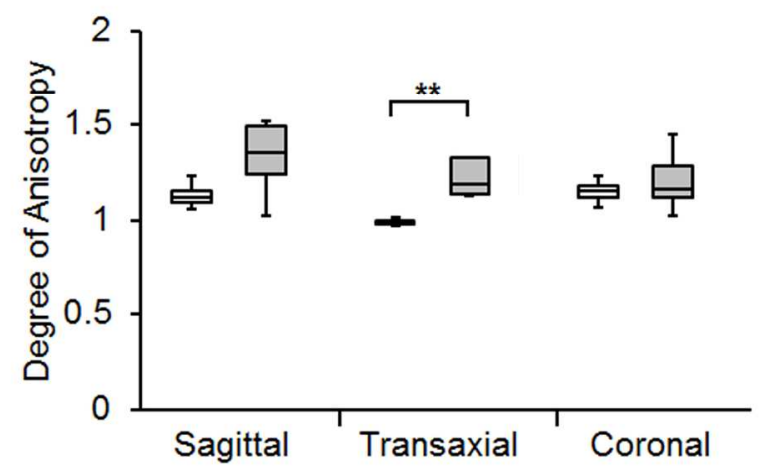

Figure 4: Box plot, reporting median values and first and third quartiles of the measurements made using micro-CT. Whiskers represent the maximum and minimum values. (a) Distance measurements taken along the $x$-axis (See figure 2) in the sagittal, transaxial and coronal views, for normal and distorted implants. (b) Distance measurements taken along the $y$ axis in the three views. There was a significant decrease in all views. (c) The degree of anisotropy calculated for the sagittal $(D A=y / x)$, transaxial $(D A=x / y)$ and coronal $(D A=y / x)$ views. $* p<0.05, * * p<0.01$, Mann-Whitney U-test.

$$
141 \times 250 \mathrm{~mm}(300 \times 300 \mathrm{DPI})
$$

John Wiley \& Sons, Inc.

This article is protected by copyright. All rights reserved. 


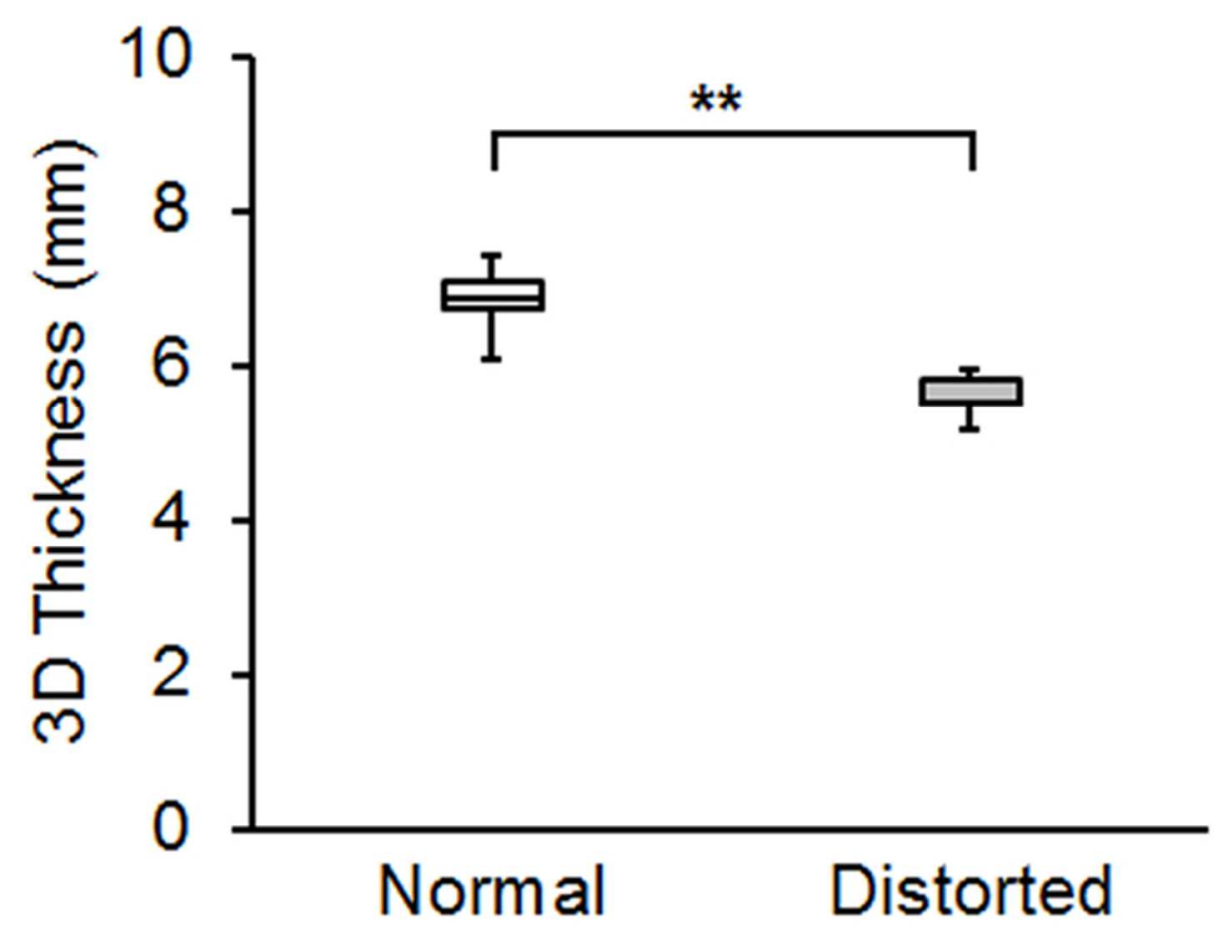

Figure 5: Box plots, reporting median values and first and third quartiles of 3D-thickness measurements made using micro-CT. Whiskers represent the maximum and minimum values. There was a significant decrease in the 3D-thickness of distorted implants compared with normal implants. **p<0.01, MannWhitney U-test.

$59 \times 44 \mathrm{~mm}(300 \times 300$ DPI $)$

John Wiley \& Sons, Inc.

This article is protected by copyright. All rights reserved. 


\section{University Library}

\section{- M M N E R VA A gateway to Melbourne's research publications}

Minerva Access is the Institutional Repository of The University of Melbourne

Author/s:

Anderson, EE;Perilli, E;Carati, CJ;Reynolds, KJ

Title:

Quantifying shape changes of silicone breast implants in a murine model using in vivo micro-CT

Date:

2017-08-01

Citation:

Anderson, E. E., Perilli, E., Carati, C. J. \& Reynolds, K. J. (2017). Quantifying shape changes of silicone breast implants in a murine model using in vivo micro-CT. JOURNAL OF BIOMEDICAL MATERIALS RESEARCH PART B-APPLIED BIOMATERIALS, 105 (6), pp.1447-1452. https://doi.org/10.1002/jbm.b.33682.

Persistent Link:

http://hdl.handle.net/11343/291184 